\title{
Context Aware Load Balancing in IoT
}

\author{
Ashok V. Sutagundar \\ Department of ECE, BEC Bagalkot -58710, INDIA \\ Email: sutagundar@gmail.com \\ Prashant Sangulagi \\ Department of ECE, BKIT Bhalki-585328, INDIA \\ Email: psangulgi@gmail.com \\ Neelamma Tarapur \\ Department of ECE, BEC Bagalkot -58710, INDIA \\ Email: neelu7tarapur@gmail.com
}

-ABSTRACT-

The IoT is the current revolt in Internet, mobile and more technologies. By adding intellect to the physical objects the objects can communicate each other to perform the desired job which reduces the human intervention. In this paper we propose a context aware load balancing in IoT. Load balancing is the splitting of traffic from one path to the other path to avoid congestion in the network. Load balancing is done by computing multipath routing, and multipath routing is computed based on context of data such as weight factor and quality of link. In this paper as the source mode initiates the route discovery by flooding the route request message to the destination in the network and waits for route reply message while migrated route packet collects the information such as node id, energy, distance, neighbor node count and mobility of nodes from all the intermediate nodes and gives it to the destination node. Once the destination receives the packet, it computes the node disjoint paths and assigns the priorities based on the type of request received. The proposed scheme is simulated for its effectiveness and the performance parameters used are path discovery time, load balancing time, throughput and packet delivery ratio.

Keywords - Load balancing, Context Aware, IoT, Multipath Routing.

\section{INTRODUCTION}

$\mathrm{T}$ he Internet of things is known to be the communication of internet with the real globe devices or things and people expands the thought from person to device communication to the device to device communication, by providing the unique address to the things or the devices, which are recognized uniquely. By adding intelligence to the physical objects, objects can communicate with other to perform the assigned job which in turn reduces the human presence. In modern years, the Internet of Things (IoT) has grown to be a important topic to the researchers across the world. it has made its method into different scopes like transportation, cultivation, commerce, and healthcare due to its quality like running in an IP based network and capable of holding millions of nodes and the nodes are capable of communicating and cooperating with each other to achieve a target .

In IoT the communication among the devices can be carried out by using the wireless networks. The remote server is used to stock up and progression the data. Because of the limitation of range, power utilization and computational potential of an IoT devices, the caching and dealing out will be constrained to some accessible resources. In IoT device routing plays a important role. Routing is a most demanding element that is considered in IoT because of its in built properties. Sometimes routing protocol explains how routing procedure takes place among the devices and communicates with one another in the network and transferring the control data to select the best routes among multiple routes [9]. In routing procedure the information has to be shared from a source node to the destination node using the nearest neighbor nodes. The best path from the source and the destination node is calculated using the routing algorithms. Authors have proposed different algorithms and protocols to increase the network lifetime, and to increase the routing efficiency. The load balancing can be used to distribute the load among all the nodes so that energy should be consumed among all nodes equally and network efficiency is also improved [10].

This paper presents context aware load balancing in Internet of Things, load balancing is the spreading of the traffic along multiple routes that reduce the packet loss in the network. Load balancing is done by computing multipath routing among the source device and the destination device. Multiple routes help in increasing the reliability of node for data transmission and throughput by means of energy consumption and bandwidth aggregation. In our work multipath routing is computed based on the context of data.

This paper is organized into following sections. Section I presents the Introduction, Section II presents the Literature Survey, Section III presents context aware load balancing in IoT, Section IV presents the proposed work, section V presents Simulation and Results, Section VI present Conclusion of the work.

\section{LITERATURE SURVEY}

Paper [1] The IoT may agree to the computerization of the whole thing surrounding us. Author has offered a 
summary of the basis of IoT idea such as its technologies, procedures, applications, and in progress to investigate, addressing the different aspects of IoT. To understand the overall architecture, and the role of the different mechanism and protocols that form the IoT. The author provides the better basis for the researchers and to the practitioners who are interested to gain an insight into the IoT technologies.

Paper [2] author has proposed to store the popular funds in the brokers and deliberate a standard measurement to quantify the status of a resource. In count, to reduce the total average delay amid the broker's author has proposed the popular resource re-stocking method. Author have considered the issues to be NP-hard and planned the LEARN algorithm to resolve the issue. Author has expressed the performance of LEARN via general simulations.

Paper [3] author has proposed the reservation of bandwidth and the control strategy based on needs of traffic for unlike QoS related services. A class-aware balancing of load and context aware policy handover was calculated to maintain the reservation of bandwidth for unlike modules and suggests the customers to make a handover to share out the traffic weight evenly all over the network. This mechanism of load balancing allows more and more users to connect with the Wi-Fi system, while by the Wi-Max as the smooth handover to uphold Quality of service/Quality of Experience. Simulation results have shown that the proposed method improves the quality of service and experience for the end users with low cost of communication.

Paper [4] in this work author proposes weight estimation method for a Li-Fi or RF hybrid IoT network using the evolutionary game theory. The Nash Equilibrium is measured as the result for the load balancing game theory and may be obtained in small duration by simulation. As Compared to the usual load balancing algorithm, the proposed evolutionary game theory algorithm can reach a superior point of customer fulfillment caused by the dynamic plan implementation of AP collection to the customers. As well, as the proposed system is executed by using the central unit and it does not require an optimized threshold, the calculation of complexity in central unit is decreased when compared to the predictable centralized methods.

\section{CONTEXT AWARE LOAD BALANCING IN IOT}

This section describes the network model, weight factor, quality of link, proposed block diagram and multipath computation.

\subsection{Network Model}

The network environment is shown in the below figure 1 . It consists of IoT devices which are randomly deployed. It also consists of gateway, cloud and virtual network. IoT devices gather the data and it sends data to the cloud through gateway. The cloud stores the data of each device in the network and stored data helps in computing the path from source to the destination device. In the virtual network actual data is not stored, just the information of the data is stored. The virtual network gives the stored information to the source device to initiate the path discovery.

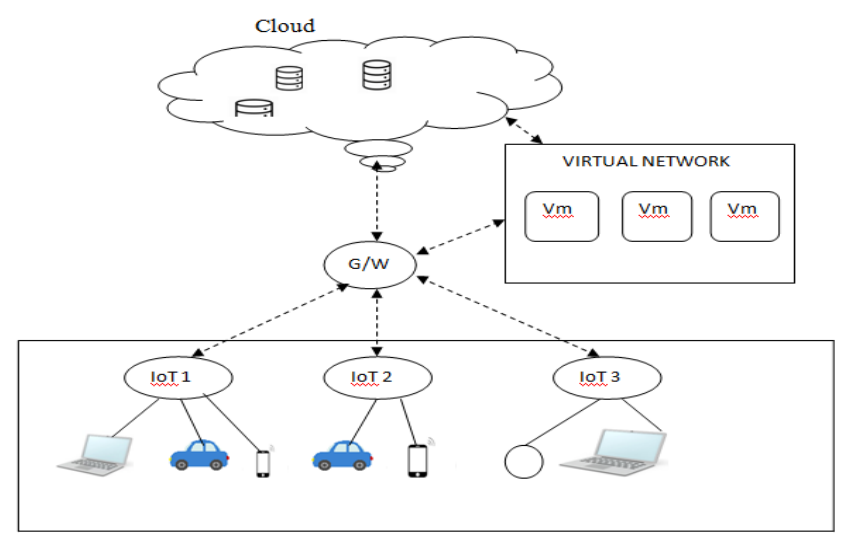

Figure 1: Network Environment

\subsection{Weight Factor}

This section presents weight factor procedure, example scenari of weight factor

The distance between the nodes is computed by equation (1).

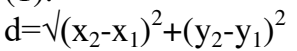

$\left(\mathrm{x}_{1}, \mathrm{y}_{1}\right)$ are the co ordinate of node 1 and $\left(\mathrm{x}_{2}, \mathrm{y}_{2}\right)$ are the coordinates of the node

The residual energy is computed by equation (2).

$\mathrm{E}_{\mathrm{RES}}=$ Initial Energy - Current Energy-----

Consider every node in the scenario computes its weight factor. The weight factor can be computed by using the following procedure

$\mathrm{W}_{\mathrm{f}}=\left(\mathrm{E}_{\mathrm{RES}} \times \mathrm{N}_{\mathrm{c}} \times \mathrm{B}_{\mathrm{n}}\right) /\left(\mathrm{M}_{\mathrm{b}} \times \mathrm{d}\right)$

$\mathrm{W}_{\mathrm{f}}=\mathrm{K}\left(\mathrm{E}_{\mathrm{RES}} \times \mathrm{N}_{\mathrm{c}} \times \mathrm{B}_{\mathrm{n}}\right) /\left(\mathrm{M}_{\mathrm{b}} \times \mathrm{d}\right)$

Where $\mathrm{W}_{\mathrm{f}}=$ Weight factor, $\mathrm{K}=$ Proportional constant, $E_{\text {Res }}=$ Residual energy of node, $N_{c}=$ Neighbour node count, $\mathrm{B}_{\mathrm{n}}=$ Bandwidth of node, $\mathrm{M}_{\mathrm{b}}=$ Mobility of nodes, $\mathrm{d}=$ Distance between nodes with respect to the destination. In Eqn[3] the Weight factor $\left(\mathrm{W}_{\mathrm{f}}\right)$ is directly proportional to the residual energy of the node, bandwidth of the node and neighbour node count and weight factor is inversly proportional to the mobility of the nodes and distance between the nodes.

3.3 Example Scenario

Let us consider $\mathrm{W}_{1}, \mathrm{~W}_{2}, \mathrm{~W}_{3}, \mathrm{~W}_{4} \ldots \ldots . \mathrm{W}_{\mathrm{n}}$ are the weight assigned to each device in the path.The highest weight factor is given the first priority to send the data through it.

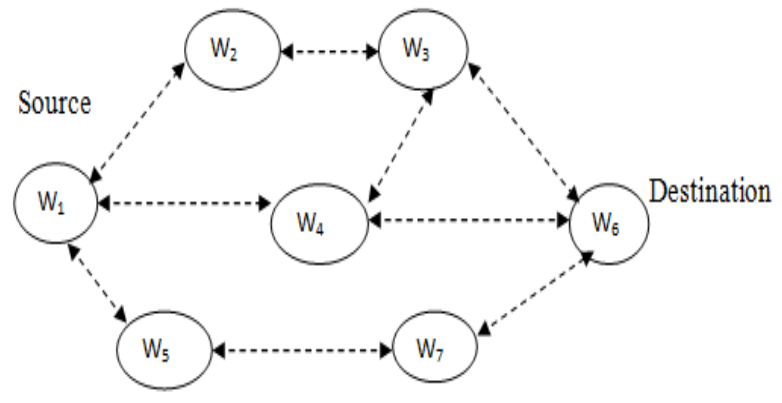

Fig. 2: Weight factor 
The weight factor of each node i.e W1, W2, W3, W4, W5, W6 are calculated using equation [2].

The total weight factor of path one (Wp1) is Wp1=Sum (W1, W2, W3, W6) --The total weight factor of path two (Wp2) is $\mathrm{Wp} 2=$ Sum $(\mathrm{W} 1, \mathrm{~W} 4, \mathrm{~W} 6)$

The total weight factor of path three (Wp3) i

Wp3=Sum(W1,W4,W3,W6)

The highest priority of weight factor is given by

$\mathrm{Pwf}=\max (\mathrm{Wp} 1, \mathrm{Wp} 2, \mathrm{Wp} 3-------\mathrm{Wpn})$

$\mathrm{Pw}_{\mathrm{f}}=$ priority of weight factor

\subsection{Quality Link}

The link superiority is evaluated among the source and destination node. Quality of link depends on the node capacity and different technologies used by the nodes.

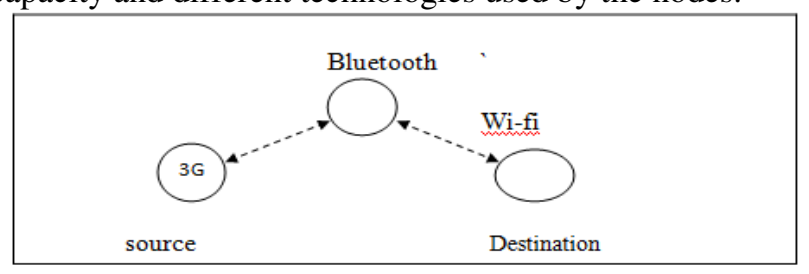

Fig. 3: Heterogeneous links case 1

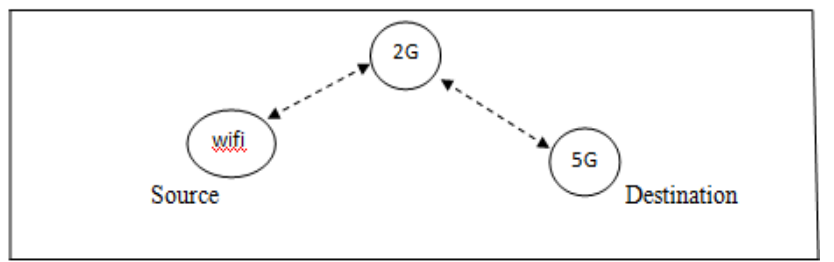

Fig. 4: Heterogeneous links case 2

Case 1: As shown in the figure 3 source node uses the 3G network to communicate with next hop node, and hop node uses the Bluetooth to communicate the data. As the $3 \mathrm{G}$ network has high speed compared to the Bluetooth and $3 \mathrm{~g}$ network can communicate for long distance and Bluetooth can communicate only for around 30 meters, so when source node sends data to the next node there will be packet loss due to the congestion in the link. Since source node has high speed compared to hop node ,the source node send data packets continuously but due to the less speed of hop node it cannot control the traffic and there will be loss of data in the network.

Case 2: As shown in the figure 4 heterogeneous links connected to the Wi-Fi, 2G, and $5 \mathrm{G}$ networks .As the source node uses the Wi-Fi technology to send data and hop node uses $2 \mathrm{~g}$ technologies to send the date so there will be compatibility in the speed between the node and there is no loss of packet in the case 2. So the quality of the link is good to communicate.

\subsection{Multipath Computation}

Multipath route computation scenario is shown in the figure 5 .

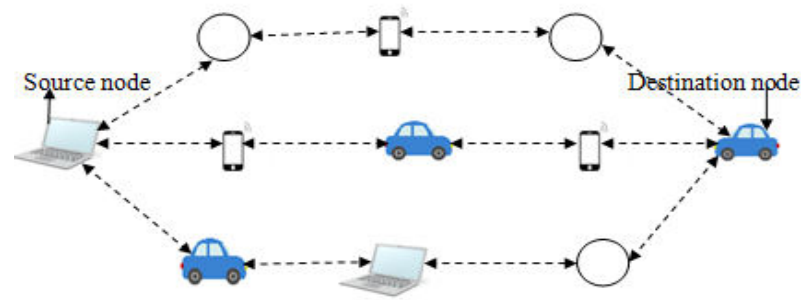

Fig. 5: Multipath Computation

As the cloud has information such as node ID, node location, mobility of node, node energy, and neighbor node count of all intermediate nodes, the source node collects the information from the virtual network and it computes the multipath routing .The next hop is selected based on the energy, bandwidth, mobility, and neighbor node count of the node. In our work we consider weight factor to discover the reliable path to the destination. Weight factor is weight assigned to each device in the network. Here the weight factor depends on the node information of each intermediate node. The highest weight factor is given the first priority to send the data through it. The multipath routing is computed using the priority weight factor of the path. Source node performs the path discovery by collecting the information from virtual network, information such as node energy, node bandwidth, node ID, mobility of node, node location. out of the existing route the destination node computes one of the route based on the context of the node . the context we considered in our project are Weight factor of node and Quality of link.

\section{PROPOSED WORK}

As shown in the figure 6 different devices are deployed randomly over the entire network environment.

In our proposed work the devices we deployed are pc, mobile phones, vehicles and sensor nodes etc--. Each device in the network has their respective gateways. Mobile networks has the gateway as base station, road side unit as gateway for vehicular networks, sink node for sensor nodes and routers for pc. The devices can also communicate with the gateway of other devices to know the information of particular device. Each devices in the network are connected to one another and each gateway is connected to the cloud and cloud in turn connected to the virtual network. First step is to find the location of destination node, in this source node finds the location of the destination node. If the destination device exists within the communication range of source device then it directly find the location of the destination device. If destination device is not within the communication range of source device, then the source node uses the intermediate devices to find the location of the destination device. Source device communicate with the gateway of that particular destination node, if the destination node is not located in that particular gateway then the gateway of the destination node communicate with the cloud to find the location of destination node. Cloud has the information of all nodes, such as node ID of every intermediate node 
Location, node Energy, node Bandwidth, mobility of node, neighbor node Count.

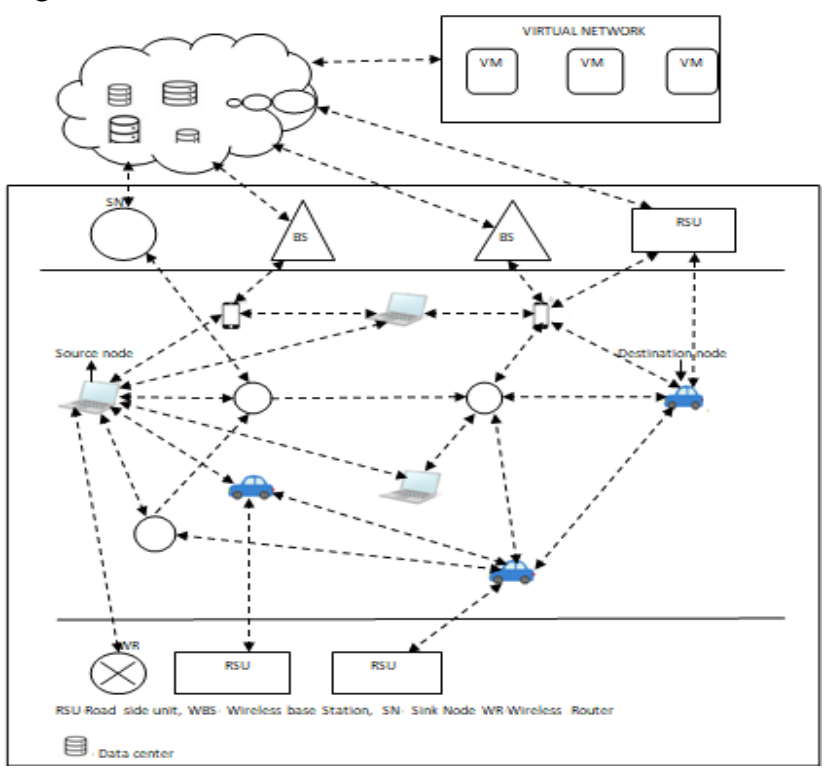

Fig. 6: Proposed block diagram

The cloud checks for particular location of the destination node and it sends to the source node. Once the location of the destination node is found then the multipath routing is computed between the source and destination node

\section{SIMULATION AND RESULTS}

The simulation of proposed work is done by using Network Simulator-2.

5.1 Simulation Inputs: Some of the simulation inputs are as shown in Table 1. The devices are mobile in nature. And whenever they have an update then efficient multipath routing is considered based on the type of data they are intended to send. If the path selected is having high traffic then alternative suitable path is considered based on the type of data to be transferred and hence the load is balanced by selecting the efficient path to destination. Various iterations have been considered to check the efficiency of the proposed work and they are analyzed with many performance parameters.

TABLE 1: Simulation Parameters

\begin{tabular}{|c|c|c|}
\hline Parameter & Notations & Value \\
\hline Number of devices & $\mathrm{N}$ & 300 \\
\hline Source node & $\mathrm{S}_{\mathrm{n}}$ & 14 \\
\hline Destination node & $\mathrm{D}_{\mathrm{n}}$ & 20 \\
\hline Propagation constant & $\mathrm{K}$ & 2 \\
\hline Mobility range & $\mathrm{Mb}_{\mathrm{r}}$ & $30 \%$ to $50 \%$ \\
\hline $\begin{array}{c}\text { Communication } \\
\text { range }\end{array}$ & $\mathrm{R}_{\mathrm{x}}$ & $500 \mathrm{mts}$ \\
\hline
\end{tabular}

5.2 Performance Parameters: Simulation of Proposed Work is estimated by means of following performance parameter which is described below.
1. Route discovery time: It is time taken with the source device to discover the path to the destination. The proposed work consumes very less time to find the destination when compared with ant based routing. The ant based routing is also type of multipath routing but it consumes lots of time and energy to find the route and sending data to destination. The route discovery time by the proposed work when compared to ant based routing is as shown in figure 7 . When node density becomes high then the time taken by the ant based routing is becoming high where as proposed work also consumes time but in small manner.

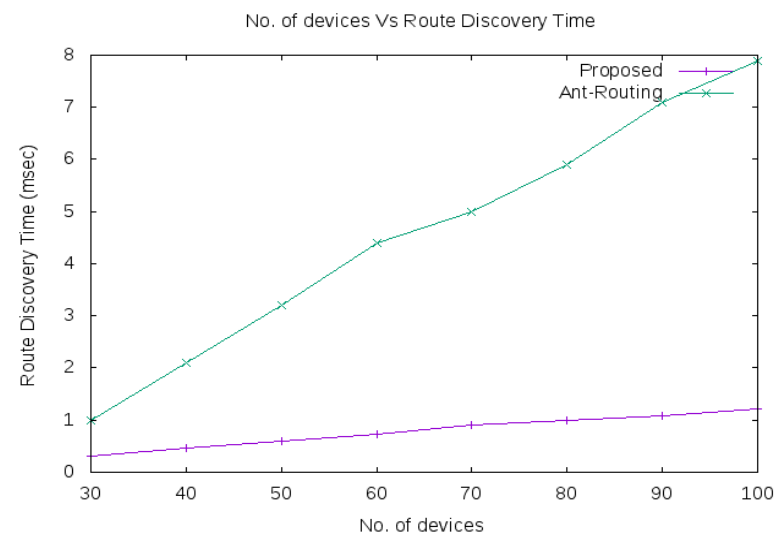

Fig. 7: Number of devices vs. Route discovery time

2. Packet Delivery Ratio: It is defined as the ratio of total number of packets generated from the devices to the number of packets received at the destination. The amount of packet dropped in the proposed methodology is very less due to efficient usage of load balancing technique which was not present in the ant based and hence lots of packets are dropped while travelling from source point to destination point. The packet delivery ration vs number of devices is shown in figure 8 . The packet number increases with more devices inclusion in the network but proposed work efficient transfer maximum packets to destination with very less number of packets are dropped while reaching to destination.

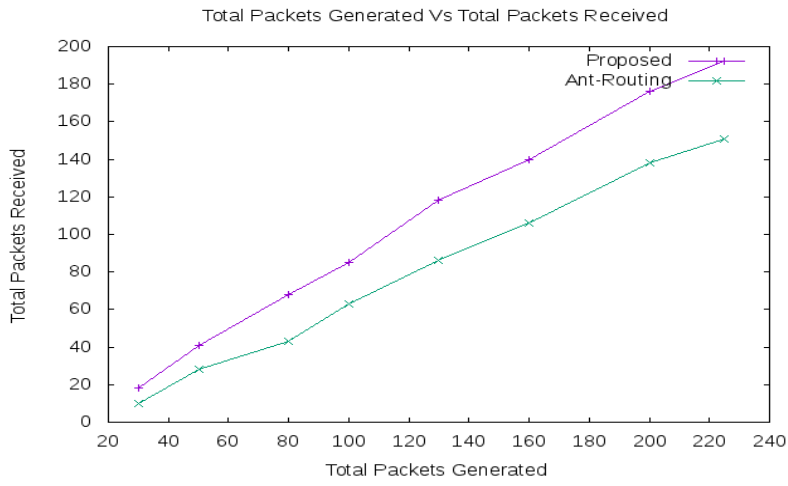

Fig 8: Number of devices vs. Packet delivery ratio

3. Delay: Delay is the total time taken by the whole system to complete one full task starting from source node. Time is consumed to find the route from source to destination i.e device to cloud server. Simultaneously more time is 
consumed if size of data and number of nodes are more. From graph it is shown that the time consumed by the ant routing is high compared to proposed methodology.

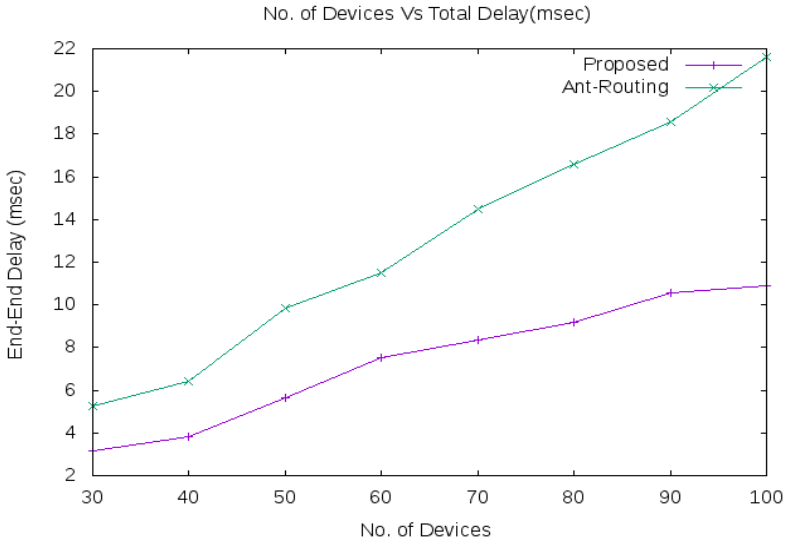

Fig. 9: Number of devices Vs Total Delay

4. Load balancing: The load balancing parameter is used to check the load distribution among nodes. In ant based routing, one time defined routing path and large amount of energy is consumed which are active in the routing process and other devices will be in stationary mode. The mismatch is avoided by the inclusion of load balancing. The multipath routing is used to balance the load and made to utilize every device during transmission and reception. The figure 10 shows the load balancing approach for the proposed work compared with ant routing. The load balancing percentage is best for proposed work and not up to the mark for ant based routing.

\section{CONCLUSION}

In this paper, we presented context aware load balance in IoT. We have proposed multipath routing for balancing the load. The path from source to destination is considered based on the type of data and its priority. Based on the weighting factor the next node is selected and data is passed through it. The results shows that, the proposed work improves packet delivery ratio, response time, load balancing capacity and less delay compared to existing techniques. The efficiency of the overall network is also better in all scenarios.

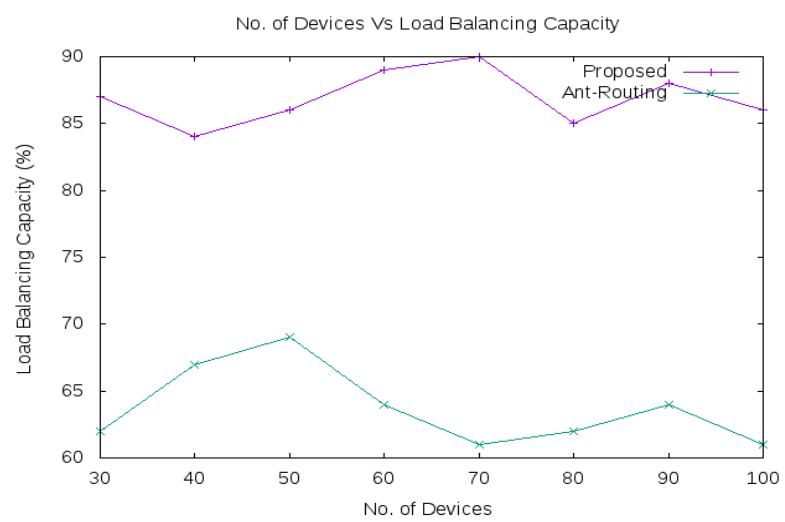

Fig. 10: Number of devices vs. Load Balancing Capacity

\section{ACKNOWLEDGEMENTS}

The research was supported by All India Council for Technical Education (AICTE) for $t$ carrying out the project "Resource Management in Internet of Things" [Ref. No. File No. 8-40/RIFD/RPS/POLICY1/2016-17 dated August 02, 2017] and also the Basaveshwar Engineering College, India for doing the work.

\section{REFERENCES}

1. Ala Al-Fuqaha, IOT: A survey on enabling technologies, protocols and applications, IEEE communication surveys and tutorials, Fourth quarter 2015, 17(4), 2347-2376.

2. X. Sun and Nirwan Ansari, Traffic load balancing among brokers at IOT application layer, IEEE Transaction on network and service management, 15(1), 2018, 489502.

3. Abhijit Sarma, Deciding handover points based on context aware load balancing in a WiFi-WiMax heterogeneous network environment", IEEE Transaction on vehicular technology, 65, 2016, 348-357.

4. Y. Wang, D. A. Basnayaka, X. Wu and H. Haas, Optimization of Load Balancing in Hybrid Li-Fi/RF Networks, IEEE Transactions on Communications, 65 (4), 2017, 1708-1720.

5. Xiang Sun, Dynamic source caching in the IOT application for smart cities, IEEE internet of things journal, 5, 2017, 1-8.

6. T. Deepa and D. Cheelu, A comparative study of static and dynamic load balancing algorithms in cloud computing, 2017 International Conference on Energy, Communication, Data Analytics and Soft Computing (ICECDS), Chennai, 2017, 3375-3378.

7. H. S. Altwassi, Z. Pervez, K. Dahal and B. Ghaleb, The RPL Load Balancing in IoT Network with Burst Traffic Scenarios, $201812^{\text {th }}$ International Conference on Software, Knowledge, Information Management \& Applications (SKIMA), Phnom Penh, Cambodia, 2018, 17.

8. Hyung-Sin kim, Load Balancing Under Heavy Traffic in RPL Routing Protocol for Low Power and Lossy Networks, IEEE Transactions on Mobile Computing, 16(4), 2017, 964-979.

9. Koffka Khan, Wayne Goodridge, Link-and NodeDisjoint Evaluation of the Ad Hoc on Demand Multi-path Distance Vector (AOMDV) Routing Protocol in Wireless Sensor Networks, Int. J. Advanced Networking and Applications, 10(01), 2018, 3674-3686.

10. Rupinder Kaur, Dr.Kanwalvir Singh Dhindsa, Efficient Task Scheduling using Load Balancing in Cloud Computing, Int. J. Advanced Networking and Applications, 10(03) 2018, 3888-3892. 